\title{
LUNG CANCER
}

\section{Squiring immunotherapy to CheckMate}

In patients with advanced-stage squamous non-small-cell lung cancer (NSCLC) there remains a high unmet need to improve outcomes: over the past two decades, no new standard-of-care therapy has been approved. Now, two phase III studies of two different targeted agents for NSCLC show great promise in turning this trend around.

Necitumumab, a second-generation anti-EGFR monoclonal antibody, was shown to be well tolerated in a phase I study. This finding prompted Nicholas Thatcher and coauthors to conduct the open-label, randomized phase III SQUIRE trial comparing necitumumab plus gemcitabine and cisplatin versus gemcitabine and cisplatin alone in patients with previously untreated stage IV squamous NSCLC.

Patients with extensive metastatic disease who were assigned to the necitumumab combination had an improved overall survival (11.5 months versus 9.9 months) and corresponding significant improvement in progression-free survival (PFS).

Thatcher puts these impressive results in context "SQUIRE was the first randomized phase III trial to show that the addition of a targeted agent to a platinum-based doublet improves survival in the first-line treatment of advanced-stage squamous NSCLC. These results confirm the benefit of the addition of an anti-EGFR antibody to standard chemotherapy in this setting, and represent clinically meaningful progress in the treatment of squamous NSCLC."

In the second study, CheckMate 017, 272 patients with stage IIIB or IV squamous NSCLC were randomly assigned to secondline therapy with docetaxel or the anti-PD-1 antibody nivolumab. Impressively, overall survival was significantly improved with nivolumab (9.2 months versus 6.0 months); moreover, the overall survival rate at 1 year was almost twice as high for nivolumab compared with docetaxel ( $42 \%$ versus 24\%). The median PFS was also improved with nivolumab. Importantly, significantly fewer grade 3 adverse events were observed with nivolumab compared with docetaxel (7\% versus 55\%). Collectively, these results point to new standards of care in this patient population.

\section{Lisa Hutchinson}

Original articles Thatcher, N. et al. Necitumumab plus
gemcitabine and cisplatin versus gemcitabine and
cisplatin alone as first-line therapy in patients with
stage IV squamous non-small-cell lung cancer (SQUIRE):
an open-label, randomised, controlled phase 3 trial.
Lancet Oncol. doi:10.1016/S1470-2045(15)00021-2 |
Brahmer, J. et al. Nivolumab versus docetaxel in advanced
squamous-cell non-small-cell lung cancer. N. Engl. J. Med.
doi:10.1056/NEJMoa1504627

